\title{
A Note on Lienard-Chipart Criteria and its Application to Epidemic Models
}

\author{
Auni Aslah Mat Daud \\ Faculty of Ocean Engineering Technology and Informatics, Universiti Malaysia Terengganu, Kuala Nerus, 21030, Malaysia
}

Received October 12, 2020; Revised January 7, 2021; Accepted January 20, 2021

\begin{abstract}
Cite This Paper in the following Citation Styles
(a): [1] Auni Aslah Mat Daud, "A Note on Lienard-Chipart Criteria and its Application to Epidemic Models," Mathematics and Statistics, Vol. 9, No. 1, pp. 41-45, 2021. DOI: 10.13189/ms.2021.090107.

(b): Auni Aslah Mat Daud, (2021). A Note on Lienard-Chipart Criteria and its Application to Epidemic Models. Mathematics and Statistics, 9(1), 41-45. DOI: 10.13189/ms.2021.090107.
\end{abstract}

Copyright $@ 2021$ by authors, all rights reserved. Authors agree that this article remains permanently open access under the terms of the Creative Commons Attribution License 4.0 International License

\begin{abstract}
An important part of the study of epidemic models is the local stability analysis of the equilibrium points. The linear algebra method which is commonly employed is the well-known Routh-Hurwitz criteria. The criteria give necessary and sufficient conditions for all of the roots of the characteristic polynomial to be negative or have negative real parts. To date, there are no epidemic models in the literature which employ Lienard-Chipart criteria. This note recommends an alternative linear algebra method namely Lienard-Chipart criteria, to significantly s implify the 1 ocal s tability analysis of epidemic models. Although Routh-Hurwitz criteria is a correct method for local stability analysis, Lienard-Chipart criteria have advantages over Routh-Hurwitz criteria. Using Lienard-Chipart criteria, only about half of the Hurwitz determinants inequalities are required, with the remaining conditions of each set concern with only the sign of the alternate coefficients of the characteristic polynomial. The Lienard-Chipart criteria are especially useful for polynomials with symbolic coefficients, as the determinants are usually significantly more complicated than original coefficients as degree of the polynomial increases. LienardChipart criteria and Routh-Hurwitz criteria have similar performance for systems of dimension five or less. Theoretically, for systems of dimension higher than five, verifying Lienard-Chipart criteria should be much easier than verifying Routh-Hurwitz criteria and the advantage of Lienard-Chipart criteria may become clear. Examples of local stability analysis using Lienard-Chipart criteria for two recently proposed models are demonstrated to show the advantages of simplified Lienard-Chipart criteria over RouthHurwitz criteria.
\end{abstract}

Keywords Lienard-Chipart Criteria, Routh-Hurwitz Criteria, Local Stability Analysis, Epidemic Models

\section{Introduction}

In the study of dynamical systems, the stability analysis provides information on the behavior of solutions of differential equations. Without having to explicitly solve the system of governing equations analytically or numerically, the stability analysis describes the trajectories of dynamical systems under perturbations of initial conditions. A major part in the qualitative study of deterministic epidemiology models of populations is local stability analysis of the equilibrium points of a system. It characterizes whether nearby initial conditions will remain close to the equilibrium point or repelled away from it. The equilibrium point is said to be stable if and only if all of the roots of the characteristic polynomial have negative real part.

The linear algebra method which is commonly employed in the local stability analysis is the well-known Routh-Hurwitz $(\mathrm{RH})$ criteria. In 1876, the Routh test is proposed by English mathematician Edward John Routh to determine whether all roots of the characteristic polynomial of a linear system have negative real parts. In 1895, German mathematician Adolf Hurwitz independently proposed the arrangement of the coefficients of the polynomial in a square matrix, called the Hurwitz matrix. He showed that the polynomial is stable if and only if all determinants of its principal submatrices are positive. The two procedures are equivalent, but the Routh test provides a more efficient way of computing the Hurwitz determinants than computing them directly. The criteria give conditions that must be satisfied for the equations of a linear system to have only stable solutions, without solving the system directly. The criteria provide necessary and sufficient conditions for all roots of the 
characteristic polynomial (with real coefficients) to be negative or have negative real parts.

$\mathrm{RC}$ criteria is used for local stability analysis in many applications such as infectious diseases [1, 2, 3, 4], noncommunicable diseases [5, 6, 7], spread of rumour [8], computer viruses [9], smoking [10], alcoholism [11], rotor-bearing system [12], drug transmission [13] and several branches of engineering [14]. While RH stability criteria is well known and commonly used in many studies on stability problem, LienardChipart (LC) criteria is not widely known [14] and often not included in the stability analysis [15]. To the best of my knowledge, there are no epidemic models in the literature which employ LC criteria. Although RH criteria is a correct method for local stability analysis, LC criteria possesses certain advantages over the RH criteria.

The aim of this note is to provide an alternative method namely LC stability conditions which can significantly simplify the local stability analysis of epidemic models. The proposed idea will be demonstrated using recently developed epidemic models. In Section 2, a brief introduction on LC criteria is provided and a comparison between $\mathrm{RH}$ criteria and LC criteria will be discussed. The examples of how LC criteria can help simplifying the local stability analysis of two epidemic models are provided in Section 3.

\section{The Lienard-Chipart (LC) criteria}

The LC criteria is a standard tool to understand the Hurwitz stability problem, which in turn has important consequences on the dynamics of some systems of differential equations. According to LC theorem, the system having the characteristic polynomial $P(\lambda)=C_{n}+C_{n-1} \lambda+\ldots+\lambda^{n}=0$ is stable if any of the following four sets of conditions is satisfied [16]:

1. the even coefficients and the even order Hurwitz determinants are positive.

2. the even coefficients and the odd order Hurwitz determinants are positive.

3. the odd coefficients and the even order Hurwitz determinants are positive.

4. the odd coefficients and the odd order Hurwitz determinants are positive

Following their concern with the redundancy of the RH stability conditions, the French mathematicians Lienard and Chipart established the above stability criteria in 1914 [17]. The criteria are stated in four different sets, any one of which is necessary and sufficient. They streamlined the RH criteria, showing that only about half of the Hurwitz determinants inequalities are required, with the remaining conditions of each set concern with only the sign of the alternate coefficients of the characteristic polynomial [18]. The LC criteria for polynomials of degree 1 to 12 are stated in Table 1, which displays alternating patterns for coefficients and determinants $\left(\left|H_{n}\right|\right.$ are Hurwitz determinants). Note that the LC criteria can be stated using both odd
Table 1. Lienard-Chipart criteria of $n \leq 12$

\begin{tabular}{|l|l|}
\hline $\begin{array}{l}\text { Order } \\
n\end{array}$ & Stability Conditions \\
\hline 1 & $C_{1}$ \\
\hline 2 & $C_{1}, C_{2}$ \\
\hline 3 & $C_{1},\left|H_{2}\right|, C_{3}$ \\
\hline 4 & $C_{1},\left|H_{3}\right|, C_{3}, C_{4}$ \\
\hline 5 & $C_{1},\left|H_{2}\right|, C_{3},\left|H_{4}\right|, C_{5}$ \\
\hline 6 & $C_{1},\left|H_{3}\right|, C_{3},\left|H_{5}\right|, C_{5}, C_{6}$ \\
\hline 7 & $C_{1},\left|H_{2}\right|, C_{3},\left|H_{4}\right|, C_{5},\left|H_{6}\right|, C_{7}$ \\
\hline 8 & $C_{1},\left|H_{3}\right|, C_{3},\left|H_{5}\right|, C_{5},\left|H_{7}\right|, C_{7}, C_{8}$ \\
\hline 9 & $C_{1},\left|H_{2}\right|, C_{3},\left|H_{4}\right|, C_{5},\left|H_{6}\right|, C_{7},\left|H_{8}\right|, C_{9}$ \\
\hline 10 & $C_{1},\left|H_{3}\right|, C_{3},\left|H_{5}\right|, C_{5},\left|H_{7}\right|, C_{7},\left|H_{9}\right|, C_{9}, C_{10}$ \\
\hline 11 & $C_{1},\left|H_{2}\right|, C_{3},\left|H_{4}\right|, C_{5},\left|H_{6}\right|, C_{7},\left|H_{8}\right|, C_{9},\left|H_{10}\right|$, \\
& $C_{1} 1$, \\
\hline 12 & $C_{1},\left|H_{3}\right|, C_{3},\left|H_{5}\right|, C_{5},\left|H_{7}\right|, C_{7},\left|H_{9}\right|, C_{9},\left|H_{11}\right|$, \\
& $C_{1} 1, C_{12}$ \\
\hline
\end{tabular}

and even terms. Generally, the signs of the coefficients can be determined by inspection of the polynomial.

All the sets of criteria can be very easily verified for any particular value of $n$, but the proof is beyond the scope of this paper. The LC theorem was proven by many authors (see $[19,20,21,22,23])$. The equivalence of RH and LC criteria has been verified by Fujiwara [24]. The advantages of LC criteria are more evident for polynomials with symbolic coefficients, because determinants are usually significantly more complicated than original coefficients as the polynomial order increases. For numerical coefficients the advantage is marginal if the computations are performed on computers [16]. The two criteria have similar performance for lower dimensional systems. However, the LC criteria is expected to perform better for systems of dimension higher than 5 (for systems of dimension less than or equal to 5, the RH and LC criteria are coincident). Theoretically, for systems of higher dimension, verifying LC criteria should be much easier than verifying RH criteria and the advantage of LC criteria may become clear [25].

Although the LC criteria may reduce the dynamic stability problem to a calculation, problems with symbolic polynomials can produce very complicated algebraic expressions that may not be simplified further (see examples in the next section), even using computational algebra. One option is to use the simplified theorem in [26, 27] which determine stability just by checking the coefficients' instead of calculating principal minors (therefore it is computationally less costly). The theorem states that, suppose $\left|H_{2}\right|>0$ : for $n \geq 5$, if $C_{5} \geq C_{1} C_{4}$ then the characteristic polynomial $P(\lambda)$ is unstable.

\section{Examples}

To demonstrate the advantage of using LC criteria over $\mathrm{RH}$ criteria, two recently proposed epidemic models are used as examples. Note that there are many complicated equations in both examples. The purposes of stating these equations are 
twofold. Firstly, using RH criteria, the number of complicated equations will be about twice as many. It shows the advantage of LC criteria over RH criteria. Secondly, using simplified LC criteria, the number of complicated equations is five (for $n \geq$ 5). It shows the advantage of simplified LC criteria over the original version. Furthermore, in some applications, while the expression of some generic conditions on the coefficients are complicated, they may actually be physically natural, easier to evaluate or chosen by design [26].

\subsection{SEQIJR model of disease transmission $(n=$} 6)

In [28], an SEQIJR model of epidemic disease transmission which included immunization and a varying population size is studied. The study considered immunization of susceptible people $(\mathrm{S})$, quarantine $(\mathrm{Q})$ of exposed people $(\mathrm{E})$, isolation $(\mathrm{J})$ of infectious people (I), a recovered population (R), and variation in population size due to natural births and deaths and deaths of infected people. It is stated that it is not possible to obtain useful analytical tests for the real parts of eigenvalues to be negative from the six RH criteria but it is possible to check two of the six RH criteria for the real parts of the six eigenvalues to be negative.

However, the authors decided to use the two equivalent necessary conditions for the real parts of the six eigenvalues to be negative that the sum of the six eigenvalues must be negative and the product of the six eigenvalues must be positive. Using LC criteria, the local stability analysis can be performed as follows. Let $a=J_{11}, b=J_{12}, c=J_{13}, d=J_{14}, e=J_{15}, f=$ $J_{16}, g=D_{S}, h=D_{E}, j=\gamma_{1}, t=D_{Q}, l=k_{1}, m=D_{I}, n=$ $k_{2}, o=\gamma_{2}, p=D_{J}, q=\sigma_{1}, r=\sigma_{2}$ and $s=\mu$. Therefore, the characteristic polynomial of the Jacobian matrix (Eq. 23) in [28] is given by

$$
P_{1}(\lambda)=\lambda^{6}+C_{1} \lambda^{5}+C_{2} \lambda^{4}+C_{3} \lambda^{3}+C_{4} \lambda^{2}+C_{5} \lambda+C_{6},
$$

where

$$
\begin{aligned}
& C_{1}=-a+b+h+t+m+p-s, \\
& C_{2}=b g-a h+c j+d l+t(b-a+h)+(m+p)(b-a+ \\
& h+t)+s(a-b-h-t-m-p)+m p,
\end{aligned}
$$$$
C_{3}=c j(g+p)+(b g+m p)(t-s)-a h(t+m)+
$$
$b g(m+p)+c j(m-s)-a h(p+s)+t p(b-a+h)+m(t+$ $p)(b-a+h)+f l q+e(j n+l o)+d l(p-s+g+t)+t s(a-$ $b-h)+s(m+p)(a-b-h-t)$,

$C_{4}=d g l(t+p-s)+c g j(m+p-s)+t m(b g-$ $a h)+e j n(g+m-s)+e l o(g+t-s)+t p(d l+b g-a h)+$ $m p(b g-a h+c j-a t+b t+h t)+f l q(g+t+p)+f r(j n+$ $l o)+m s(a h-b g-c j+a t-b t-h t)+t s(a h-b g-$ $d l)+m p s(a-b-h-t)-p s(b g+a h-c j+a t-b t-h t-d l)$,

$C_{5}=e g(j m n+t l o-j n s-l o s)+d g(t l p-l p s-$ $t l s)+m p(c g j+b g t-a h t)+f g(t l q+l p q+j n r+l o r)+$ $f t l p q+f r(j m n+t l o)-e t l o s-c g j p s+t p s(a h-b g-d l)+$ $m s(a h t-c g j-b g t-e j n)+m p s(a h-b g-c j+a t-b t-h t)$,

$C_{6}=f g(t l p q+j m n r+t l o r)-e g(j m n s+t l o s)-$ $d g t l p s+m p s(a h t-c g j-b g t)$.

Using LC criteria, the system is locally asymptotically stable if the coefficients $C_{1}, C_{3}, C_{5}$ and $C_{6}$ are positive and the Hurwitz determinants $\left|H_{3}\right|$ and $\left|H_{5}\right|$ are positive. It shows that LC criteria can greatly reduce the computation cost (compared to $\mathrm{RH}$ criteria). However, the computation cost can be reduced even further using the aforementioned simplified version of LC criteria. That is, the coefficients that must be computed are $C_{1}, C_{2}, \ldots, C_{5}$ only. Note that $C_{1}, C_{2}$, and $C_{3}$ are needed because the simplified version of LC criteria still require $\left|H_{2}\right|=C_{1} C_{2}>C_{3}$.

\subsection{Model of Wolbachia infection $(n=9)$}

In [29], a hierarchy of reduced models was proposed and analyzed to approximate a detailed system of nine ordinary differential equations (ODEs) that describes the spread of Wolbachia infection in mosquitoes. The 9-ODE model of Wolbachia infection captures the detailed transmission dynamics and the defined model parameters are biologically relevant quantities. However, it is stated that it is difficult to analyze. As a result, its local stability is not analysed. Using LC criteria, the local stability analysis can be performed as follows. Let $a=\mu_{a}+\psi, b=\eta_{u}, c=v_{u} \eta_{w}, d=v_{w} \eta_{w}, e=$ $b_{f} \psi, f=\sigma+\mu_{f u}, g=\sigma+\mu_{f w}, h=\sigma m_{u}, j=\mu_{f u}, k=$ $\sigma m_{w}, l=\sigma, m=\mu_{f w}, n=b_{m} \psi, o=\mu_{m u}$ and $p=\mu_{m w}$. Therefore, the characteristic polynomial of the coefficient matrix of equations (2.1a)-(2.1i) in [29] is given by

$P_{2}(\lambda)=\lambda^{9}+C_{1} \lambda^{8}+C_{2} \lambda^{7}+C_{3} \lambda^{6}+C_{4} \lambda^{5}+C_{5} \lambda^{4}+$ $C_{6} \lambda^{3}+C_{7} \lambda^{2}+C_{8} \lambda+C_{9}$,

where

$C_{1}=2 a+f+g+2 j+m+o+p$,

$C_{2}=a(a+2 f+2 g)+f g+j^{2}+2 j(2 a+f+g)+$ $(m+o+p)(2 a+f+g+2 j)+m p+o m+o p$,

$C_{3}=j^{2}(2 a+f+g)+a(2 f g+a(f+g))-b e h+2 j\left(a^{2}+\right.$ $2 a f+2 a g+f g)-d e l+(m+o+p)\left(\left(a^{2}+j^{2}+2 a f+2 a g+\right.\right.$ $f g)+2 j(2 a+f+g))+o p m+(o m+m p+o p)(2 a+f+g+2 j)$,

$C_{4}=a^{2} j(j+2 p)+a^{2} f g-b e h(a+g+j+m+$ $o+p)-\operatorname{del}(a+f+2 j+o+p)+j(j m+2 m o+j p+$ $2 o p)(2 a+f+g)+a(m+o+p+2 j)(a f+a g+2 f g)+$ $2 f g j(m+o)+j(j o+2 m p)(2 a+f+g)+a(2 j m+2 j o+$ $o p)(a+2 f+2 g)+m(o+p)\left(a^{2}+j^{2}\right)+(m o+2 j p+$ $\left.m p+j^{2}\right)(2 a f+2 a g+f g) o p\left(f g+j^{2}\right)+\operatorname{mop}(2 a+f+g+2 j)$,

$C_{5}=a j(j+2 m)(a f+a g+2 f g)+a^{2} f g(2 j+m+$ $p+o)-a b e h(g+j+m+o+p)-b e h(g o+g m+g p+g j+$ 
$j m)-\operatorname{del}\left(j^{2}+a f+2 a j+2 f j\right)+j^{2}(m+o+p)\left(a^{2}+2 a f+\right.$ $2 a g+f g)+j(o+p)\left(2 a^{2} f+2 a^{2} g+4 a f g-b e h\right)-\operatorname{delo}(a+f+$ $2 j+p)+(m o+m p+o p)\left(a^{2} f+a^{2} g+2 a f g-b e h\right)-\operatorname{del}(a+$ $f+2 j)+2 f j+2 g j)+(m p+o p+m o)\left(j^{2}(2 a+f+g)+\right.$ $\left.2 j\left(a^{2}+2 a f+2 a g+f g\right)\right)+m o p\left(a^{2}+j^{2}+2 a f+2 a g+f g+4 a j\right.$, $C_{6}=a^{2} f g j(j+2 p+2 m+2 o)+a^{2} f g(m o+o p+$ $m p)-\operatorname{adel}\left(j^{2}+f p+2 j p+f o+2 j o+2 f j\right)-d e j^{2}(f l+$ $l p+l o)+\operatorname{del}(b e h-2 f j p)+a j^{2} m(a f+a g+2 f g)-$ $\operatorname{abeh}(g(m+o)+j m+o(g+m+j)+p(j+m+o))-$ $\operatorname{begh}((j+p)(a+m+o)+j p+m o)+j^{2} o\left(a^{2} f+a^{2} g+\right.$ $2 a f g)+j(j o(m o+o p+m p)+2)\left(a^{2}+2 a f+2 a g+f g\right)+$ $j m o\left(2 a^{2} g-b e h\right)+a^{2} j^{2} p(f+g)+2 a f g j\left(j p+2 g a^{2} m o\right)+$ $j p(m+o)\left(2 a^{2} f+2 a^{2} g+4 a f g-b e h\right)-\operatorname{delo}(a p+f p+$ $2 j p+2 f j)+\operatorname{mop}\left(j^{2}(2 a+f+g)+\left(a^{2} f+a^{2} g+2 a f g-b e h\right)\right)$,

$C_{7}=-a d e j^{2}(f l+l o+p)+b d e^{2} h(j l+l o)+m o p+$ $a^{2} f g j^{2}(m+p+o)-\operatorname{deflo}\left(j^{2}+2 a j\right)+a j^{2} m o(a f+a g+$ $2 f g)+j m o\left(2 a^{2} f g-b e g h\right)-a b e h(g(m o+j p+m p+j m+$ $j o+o p)+j(o p+m o+m p)+\operatorname{delp}\left(b e h-f j^{2}-2 a f j\right)+$ $\left.a j^{2} p(m+o)(a f+a g+2 f g)+j\left(2 a^{2} f+2 a^{2} g+4 a f g-b e h\right)\right)-$ gop $\left(\right.$ behj $\left.-2 a^{2} f j\right)-\operatorname{delop}\left(j^{2}+a f+2 a j+2 f j\right)+\operatorname{mop}\left(j^{2}\left(a^{2}+\right.\right.$ $2 a j+2 a g+f g)+\left(a^{2} f g-b e g h\right)+j m p\left(2 a^{2} f-b e g h\right)$,

$C_{8}=(a f j-b e h)(a g j(m o+m p+o p)-\operatorname{dejl}(o+$ $\left.p))+\operatorname{delop}\left(b e h-a j^{2}-f j^{2}-2 a f j\right)-b e h(a j+g j+a g)\right)+$ $\operatorname{mop}\left(a^{2} j^{2}(f+g)+2 a f g j(j+a)\right.$,

$C_{9}=($ dejlop - agjmop $)(b e h-a f j)$.

Using LC criteria, the system is locally asymptotically stable if the coefficients $C_{1}, C_{3}, C_{5}, C_{7}$ and $C_{9}$ are positive and the Hurwitz determinants $\left|H_{2}\right|,\left|H_{4}\right|,\left|H_{6}\right|$ and $\left|H_{8}\right|$ are positive. However, similar to example in Section 3.1, using simplified LC criteria, the coefficients required are $C_{1}, C_{2}, \ldots, C_{5}$ only. The advantages of simplified LC criteria over RH criteria are even more apparent in this example.

\section{Conclusion}

Some issues on the mathematical modeling of epidemic models have been discussed recently [7]. In this paper, the improvement on the local stability analysis of epidemic models is proposed, using the simplified version of LC stability criteria. It greatly simplifies the calculation in the analysis and reduces the computational cost. Examples of application of the LC criteria are demonstrated using two recently proposed epidemic models namely the SEQIJR model of epidemic disease transmission and a system of nine ODEs that describes the spread of Wolbachia infection in mosquitoes.

\section{Acknowledgements}

The study is part the research project Talent and Publication Enhancement-Research Grant (TAPE-RG55106) of Universiti Malaysia Terengganu.

\section{REFERENCES}

[1] T. Kuniya. Stability Analysis of an Age-Structured SIR Epidemic Model with a Reduction Method to ODEs, Mathematics, 6, 147, 2018.

[2] M. Kizito, J. Tumwiine. A mathematical model of treatment and vaccination interventions of pneumococcal pneumonia infection dynamics, Journal of Applied Mathematics, (2539465), $16,2018$.

[3] B. Gbadamosi, M.M. Ojo, S.I. Oke, M.B. Matadi. Qualitative analysis of a dengue fever model, Mathematical and Computational Applications, 23 (3), 33, 2018.

[4] M. Derouich, A. Boutayeb, EH Twizell. A model of dengue fever, BioMedical Engineering OnLine, 2:4, 2003.

[5] A. A. Mat Daud. Mathematical modeling and stability analysis of population dynamics. In: Mohd M., Abdul Rahman N., Abd Hamid N., Mohd Yatim Y. (eds) Dynamical Systems, Bifurcation Analysis and Applications, Springer Proceedings in Mathematics \& Statistics vol. 295, 3-13. Springer, Singapore, 2018.

[6] A. A. Mat Daud, C. Q. Toh, S. Saidun. A mathematical model to study the population dynamics of hypertensive disorders during pregnancy, Journal of Interdisciplinary Mathematics, 22, 433-450, 2019.

[7] A. A. Mat Daud, C. Q. Toh, S. Saidun. Development and analysis of a mathematical model for the population dynamics of Diabetes Mellitus during pregnancy, Mathematical Models and Computer Simulations, 12, 620-630, 2020.

[8] G. Chen, H. Shen, T. Ye, G. Chen, N. Kerr. A kinetic model for the spread of rumor in emergencies, Discrete Dynamics in Nature and Society, 1-8, 2013.

[9] L. X. Yang, X. F. Yang. The spread of computer viruses over a reduced scale-free network, Physica A, Vol.396, No.15, 173-184, 2014

[10] Z. Zhang, R. Wei, W. Xia. Dynamical analysis of a giving up smoking model with time delay, Advances in Difference Equations, 505, 2019. 
[11] H. F. Huo, S. H. Ma, X. Y. Meng. Modelling alcoholism as a contagious disease: A mathematical model with awareness programs and time delay, Discrete Dynamics in Nature and Society, 1, 1-13, 2015.

[12] D. A. Glasgow, H. D. Nelson. Stability analysis of rotor-bearing systems using component mode synthesis, Transactions of the ASME, Journal of Mechanical Design, 102:352-359, 1980.

[13] S. Saha, G. P. Samanta. Modelling the role of optimal social distancing on disease prevalence of COVID-19 epidemic, International Journal of Dynamics and Control, 2020.

[14] W. Squire. The Lienard-Chipart criteria for stability of polynomials, Aerospace Sciences, 2012.

[15] S. L. Wiggers, Pedersen P. Routh-Hurwitz-Liénard-Chipart Criteria. In: Structural Stability and Vibration. Springer Tracts in Mechanical Engineering. Springer, Cham, 2018.

[16] Y. S. Apte. Routh-Hurwitz stability criterion and its equivalents, IEE-IERE Proceedings India, 7, 149-154, 1969.

[17] E. I. Jury. Comments on the stability criterion of Lienard and Chipart, Linear Algebra and its Applications, 47, 169-171, 1982.

[18] Stability analysis tools. ASEN 5519-006: Fluid-Structure Interaction-Spring 2004 Aerospace Engineering Sciences, University of Colorado at Boulder, June 29, 2006.

[19] B. N. Datta. An elementary proof of the stability criterion of Lienard and Chipart, Linear Algebra and its Applications, 22, 89-96, 1978

[20] B. Anderson. The reduced Hermite criterion with application to proof of the Lienard-Chipart criterion, IEEE Transactions on Automatic Control, Vol.17, No.5, 669-672, 1972.

[21] F. R. Gantmacher. The Theory of Matrices, Vol. II,, Chelsea Publishing Co., New York, 1959.
[22] M. Marden. Geometry of Polynomials, Second Ed., American Mathematical Society: Providence, 1966.

[23] S. Barnett. A new formulation of the Liénard-Chipart stability criterion, Cambridge University Press, Proceedings of the Cambridge Philosophical Society, 269-274, 1971.

[24] M. Fujiwara. On algebraic equations whose roots lie in a circle or in a half-plane, Mathematische Zeitschrift, 24, 161-169, 1926.

[25] W. Niu, D. Wang. Algebraic approaches to stability analysis of biological systems, Mathematics and Computers in Simulation, 1, 507-539, 2008.

[26] R. Bortolatto. A note on the Lienard-Chipart criterion and roots of some families of polynomials, Research Report, Universidade Tecnologica Federal do Parana (UTFPR), Campus Londrina-PR-Brazil, arXiv:1407.4852v2 [math.DS], 2014.

[27] J. H. Kim, W. Su, Y. J. Song. On Stability Of A Polynomial, Journal of Applied Mathematics and Informatics, Vol.36, No.3-4, 231-236, 2018.

[28] S. Siriprapaiwan, E. J. Moore, S. Koonprasert. Generalized reproduction numbers, sensitivity analysis and critical immunity levels of an SEQIJR disease model with immunization and varying total population size, Mathematics and Computers in Simulation, 146, 70-89, 2018.

[29] Z. Qu, J. M. Hyman. Generating a hierarchy of reduced models for a system of differential equations modeling the spread of Wolbachia in mosquitoes, SIAM Journal on Applied Mathematics, 79, 1675-1699, 2019.

[30] A. A. Mat Daud, Some issues on the mathematical modeling of population dynamics using differential equations, International Journal of Mathematics and Computer Science, 15, 501-513, 2020 . 